\title{
New acrylate terpolymer-based nanoparticles for the release of nucleic acid: a preliminary study
}

\author{
Elena Bellotti ${ }^{1}$, Niccoletta Barbani ${ }^{1}$, Maria G. Cascone ${ }^{1}$, Caterina Cristallini ${ }^{2}$ \\ ${ }^{1}$ Department of Civil and Industrial Engineering, University of Pisa, Pisa - Italy \\ ${ }^{2}$ CNR, Institute for Polymers, Composites and Biomaterials (IPCB) UOS Pisa, Pisa - Italy
}

\begin{abstract}
Purpose: Nano-drug delivery systems based on polymeric biomaterials have received considerable interest as drug delivery vehicles. In this work, radical polymerization was carried out in order to obtain nanoparticles based on a new acrylate terpolymer (PBMA-(PEG)MEMA-PDMAEMA).

Methods: Nanoparticles were developed in the form both of nanospheres and nanocapsules, an innovative kind of hollow nanoparticles with a great potential because of their low effective density and high specific surface area. The ability of the nanoparticles to load and then release a nucleic acid (DNA) to be used in cancer treatment was also investigated.

Results: Scanning electron microscopy analysis showed a spherical shape, nanometric dimensions, and a homogeneous distribution of the nanoparticles, also confirmed by dynamic light scattering measurements. Fouriertransform infrared spectroscopy chemical imaging analysis carried out on the nanocapsules before and after removal of the core demonstrated the presence of the cavity. High-performance liquid chromatography analysis confirmed good encapsulation efficiency of DNA both for nanospheres and nanocapsules. Drug release tests showed controlled release kinetics for both the systems with a high release of DNA in the first hours. In vitro MTT assay showed that the particles do not have cytotoxic effects on the cells.

Conclusions: The preliminary investigation showed that the terpolymer-based nanoparticles developed in this study could be good candidates to be used as innovative and versatile gene delivery systems.

Keywords: Nanoparticles, Drug delivery, Nucleic acid, Hollow nanoparticles, Terpolymer
\end{abstract}

\section{Introduction}

Pharmaceutical carriers have been widely investigated in both laboratory and clinical settings to improve the efficiency of delivering therapeutic and diagnostic agents via different administration routes (1). Nano-drug delivery systems based on polymeric biomaterials have received considerable interest as drug delivery vehicles in recent decades (2). Polymeric nanocarriers are particles of a solid, spherical structure with at least 1 dimension $<500 \mathrm{~nm}(2,3)$. They are prepared from natural or synthetic polymers with a therapeutic agent encapsulated within the polymer matrix, conjugated, or absorbed onto the surface (3).

As is well known, most commonly used pharmaceuticals are not able to accumulate in the target organ or tissue in a

\section{Accepted: May 1, 2015}

Published online: September 14, 2015

\section{Corresponding author:}

Elena Bellotti

Department of Civil and Industrial Engineering

Largo Lucio Lazzarino, 2

56122 Pisa, Italy

elena.bellotti@for.unipi.it selective and quantitative way; usually they are evenly distributed within the body. Moreover, to reach the target area, the drug has to cross many biological barriers, where it can cause adverse reactions or be partially inactivated (4-5). Preclinical and clinical studies have demonstrated that drugloaded polymeric nanoparticles confer prolonged circulation time, protection of the drug from physiological degradation, enhanced accumulation in the target sites, reduced side effects, improved drug tolerance, and/or long term drug bioavailability (6).

In order to increase the amount of encapsulated drug, nanoparticles can be functionalized from a morphological point of view with the aim of obtaining a hollow structure. Hollow polymeric nanostructures have attracted considerable interest in recent years because of their new functionalities and unique physicochemical properties anticipated from polymeric materials at nanoscale level (7). Hollow spheres have great potential for promising applications because of their low effective density, high specific surface area, and many other advantageous properties (8). Thanks to their hollow core structure, these particular kinds of nanoparticles can encapsulate a large number of guest molecules such as drugs, enzymes, proteins, and genes (9). Although there are many strategies for preparing hollow polymeric spheres, a template is often needed (10). With 
the template method, hollow structures can be obtained after removing the template by dissolution, evaporation, or thermolysis. Hollow nanoparticles are often cross-linked and this offers good physical resistance, preventing their collapse during the core extraction process.

In this work, radical polymerization was carried out in order to obtain nanoparticles (NPs) based on a new acrylate terpolymer starting from butyl methacrilate, poly(ethylene glycol) methyl ether methacrylate and 2-(dimethylamino)ethyl methacrylate (PBMA-(PEG)MEMA-PDMAEMA) to be used as a platform for the delivery of a nucleic acid for cancer treatment. The idea of a new copolymer based on 3 different components is triggered by the need to combine the good properties of each element. PBMA is an acrylic polymer well known for its biocompatibility and it is often used in biomedical applications to obtain platforms for drug delivery and gene delivery (11-13). PDMAEMA has been largely utilized as a nonviral gene delivery system with low cytotoxicity (14-16). The presence of PEG chains makes the surface of the carrier more hydrophilic and minimizes the tendency to absorb proteins, thus reducing the reticuloendothelial clearance (17-19). NPs were developed both in the form of nanospheres (NSs) and nanocapsules (NCs) using the template method. The ability of the NPs to load and then release a nucleic acid to be used for different applications, including cancer treatment, was also investigated using a model molecule of DNA.

\section{Materials and methods}

\section{Preparation of nanospheres and nanocapsules}

Terpolymer-based NSs were obtained by radical polymerization starting from a diluted solution of BMA, (PEG)MEMA and DMAEMA (percentage molar ratio 80/10/10, respectively) in the presence of trimethylpropane trimethacrylate (TRIM) as cross-linker $(20 \% \mathrm{~mol} / \mathrm{mol})$. The polymerization was carried out in bidistilled water in the presence of the radical initiator $\left(\mathrm{Na}_{2} \mathrm{~S}_{2} \mathrm{O}_{5} /\left(\mathrm{NH}_{4}\right)_{2} \mathrm{~S}_{2} \mathrm{O}_{8}, 10 \% \mathrm{~mol} / \mathrm{mol}\right)$. The reaction was carried out for $3 \mathrm{~h}$, ensuring a constant stirring at $600 \mathrm{rpm}$ at $37^{\circ} \mathrm{C}$. At the end of the polymerization process, the NSs were recovered, rinsed 3 times with bidistilled water in order to remove the unreacted monomers, and then freeze-dried overnight.

NCs were obtained by an innovative technique consisting in a first step during which the PMMA NPs to be used as the core were synthesized by emulsion polymerization of methyl methacrylate in the presence of SDS. Then the polymerization of the monomers around the preformed core (PMMA core/monomers weight ratio 1:5) was carried out using the procedure previously described and subsequently the core was extracted by dispersing the NCs in chloroform (Carlo Erba Reagenti, Rodan, Italy). Finally the NCs were rinsed with bidistilled water and freeze-dried overnight. All the reagents were purchased from Sigma-Aldrich (St Louis, MO, USA).

\section{Absorption and release tests}

DNA (deoxyribonucleic acid, low molecular weight from salmon sperm, Sigma-Aldrich) was dissolved in phosphate buffered solution (PBS) to obtain a concentration of $1 \mathrm{mg} / \mathrm{ml}$. Subsequently, $10 \mathrm{mg}$ of NPs were dispersed in $1 \mathrm{~mL}$ of DNA solution and gently stirred for $2 \mathrm{~h}$. The NPs were separated from the absorption solution by ultracentrifugation (Mikro 200 Hettich Zentrifugen, Tuttlingen, Germany) at $14000 \mathrm{rpm}$ for $15 \mathrm{~min}$. A solution of DNA in PBS $(1 \mathrm{mg} / \mathrm{ml})$ was centrifuged in the same conditions and subsequently analyzed by high performance liquid chromatography (HPLC; Series 200; Perkin Elmer, Waltham, MA, USA) in order to verify the possible presence of pelleted DNA. Release tests were carried out by dispersing $10 \mathrm{mg}$ of DNA-loaded NPs into $1 \mathrm{~mL}$ of PBS at $37^{\circ} \mathrm{C}$. An aliquot of the release medium was collected at predetermined intervals, and fresh PBS was reinstated. Absorption and release tests were performed in triplicate both on the NSs and NCs. The collected samples were subsequently analyzed by HPLC.

\section{Cytotoxicity test}

To evaluate the cytotoxic characteristics of the produced NPs, the 3-(4,5-dimethylthiazol-2-yl)-2,5-diphenil tetrazolium bromide (MTT) assay was performed using 3 T 3 cells from a mouse fibroblast cell line (Cell bank Interlab Cell Line Collection; IST, Genoa, Italy). NPs were extracted in Dulbecco's Eagle's Modified Medium (DMEM, Sigma-Aldrich) $(20 \mathrm{mg} / \mathrm{ml})$ for $24 \mathrm{~h}$ at $37^{\circ} \mathrm{C}$. The cells were seeded onto 48-well plates at 2 different densities: $1.2 \cdot 10^{4}$ cells $/ \mathrm{cm}^{2}$ for the $24 \mathrm{~h}$ and $0.6 \cdot 10^{4}$ cells $/ \mathrm{cm}^{2}$ for the $72 \mathrm{~h}$-exposure period test. Then, $24 \mathrm{~h}$ after seeding, culture medium was removed from the wells and replaced with fresh culture medium $(800 \mu \mathrm{L})$ added with $200 \mu \mathrm{L}$ of sterile filtered NC and NS extract. For each extract 10 replicates were performed. No extracts were added to 12 wells used as negative controls (NC); 4 wells containing only cell culture medium were used as blank. The plates were incubated in a humidified atmosphere containing $5 \% \mathrm{CO}_{2}$ at $37^{\circ} \mathrm{C}$. After $24 \mathrm{~h}$ and $72 \mathrm{~h}$ of incubation, respectively, cell viability was measured by MTT assay as follows. A total of $100 \mu \mathrm{L}$ of MTT solution ( $10 \% \mathrm{v} / \mathrm{v}$ with respect to the culture medium volume) were added to each well. After $4 \mathrm{~h}$ incubation at $37^{\circ} \mathrm{C}$, the medium was completely removed and replaced with $1 \mathrm{~mL}$ of DMSO. After $5 \mathrm{~min}$ of slow agitation the absorbance was read at $570 \mathrm{~nm}$ using a UV spectrophotometer (V-530; Jasco, Easton, MD, USA).

\section{Characterization of the nanoparticles}

The morphology of the NPs was examined by scanning electron microscope (SEM) (JSM 5600; JEOL, Tokyo, Japan). The samples were placed on the SEM sample stage and sputter coated with gold. The mean size and polydispersity index (PDI) of the NPs dispersed in different media (ethanol, PBS, buffered solution with $\mathrm{pH} 4.00$ and 9.21) were determined by dynamic light scattering (DLS) using a Zetasizer nano ZS90 (Malvern Instruments, Malvern, UK). DLS analysis were performed in triplicate on both systems.

Fourier-transform infrared (FTIR) chemical imaging analysis was carried out in order to obtain information regarding the composition of the NPs, the removal of the PMMA core and the presence of DNA after absorption tests. HPLC 

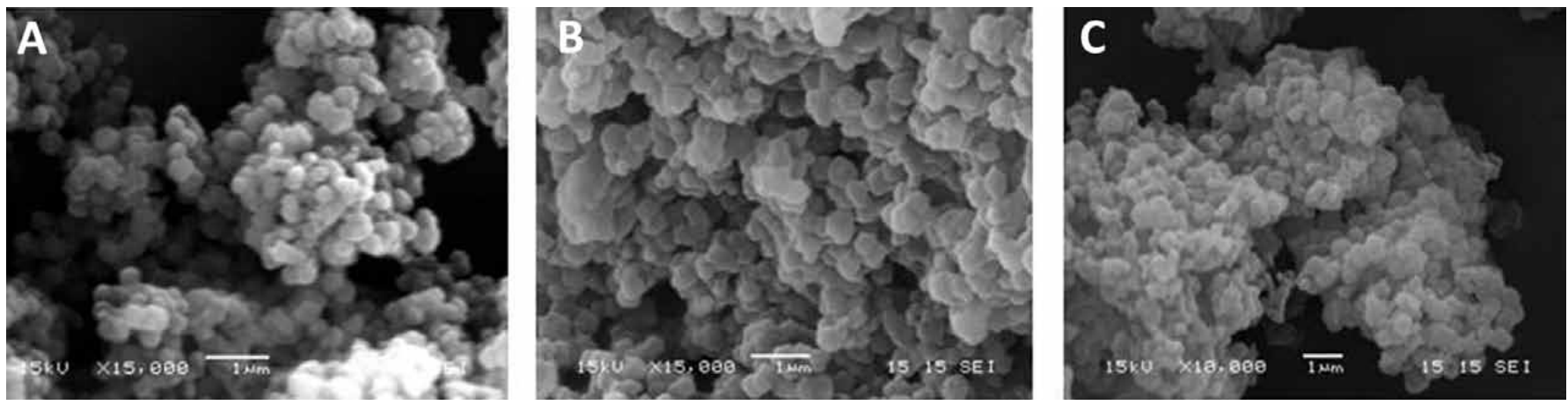

Fig. 1 - SEM analysis of NPs. (A) SEM image of NSs; (B) SEM image of NCs before and (C) after extraction of the core.

analysis was carried out to evaluate monomer conversion, using an Alltech Alltima C18 $5 \mathrm{u}(250 \mathrm{~mm} \times 4.6 \mathrm{~mm}$; Fisher Scientific, Waltham, MA, USA) as a column, and acetonitrile $(A C N) /$ water $(80 / 20 \mathrm{v} / \mathrm{v})$, fluxed at $1 \mathrm{ml} / \mathrm{min}$ as the internal mobile phase. The injected volume was $50 \mu \mathrm{L}$ and the UV wavelength was $210 \mathrm{~nm}$.

The final monomer conversion was evaluated using the following equation:

$$
x(t)=\frac{\left(C_{o}-C_{t}\right)}{C_{0}}
$$

where $\mathrm{C}_{0}$ and $\mathrm{C}_{t}$ are respectively the initial and final monomer concentration in the reactive mass.

HPLC analysis was also performed to investigate the amount of absorbed and released DNA. Samples were analyzed using a Synergi 4u Hydro-RP C18 (80 ̊, $250 \mathrm{~mm} \times 3.00 \mathrm{~mm}$; Phenomenex, Torrance, CA, USA) as a column and ACN/water $(7 / 93 \mathrm{v} / \mathrm{v})$, fluxed at $1 \mathrm{ml} / \mathrm{min}$ as the internal mobile phase. The injected volume was $50 \mu \mathrm{L}$, and the chromatograms collected at the UV wavelength of $260 \mathrm{~nm}$ were used for quantitative analysis. Released kinetics were analyzed using the semi empirical equation of the power low to describe drug release from polymeric systems:

$$
\frac{M_{t}}{M_{\infty}}=k t^{n}
$$

where $M_{t}$ and $M_{\infty}$ are the absolute cumulative amounts of drug released at time $t$ and infinite time respectively, $k$ is a constant incorporating structural and geometric characteristics of the device and $\mathrm{n}$ is the release exponent, indicative of the drug release mechanism (20).

\section{Results}

\section{Monomer conversion}

The radical polymerization of monomers in diluted condition allowed the formation of NPs directly in the reaction phase. Final conversion of the 3 monomers was greater than $95 \%$ both for the NSs and NCs.
TABLE I - Average radius and polydispersity index of nanospheres and nanocapsules

\begin{tabular}{lcccc}
\hline Dispersant & \multicolumn{2}{c}{ NSs } & \multicolumn{2}{c}{ NCs } \\
\hline & $\begin{array}{c}\text { Average } \\
r(n m)\end{array}$ & $P D I$ & $\begin{array}{c}\text { Average } \\
r(n m)\end{array}$ & $P D I$ \\
pH 9.21 & $218.9 \pm 21.2$ & 0.61 & $198.0 \pm 20.4$ & 0.68 \\
Ethanol & $193.0 \pm 20.9$ & 0.60 & $214.7 \pm 19.7$ & 0.63 \\
PBS & $188.8 \pm 17.4$ & 0.53 & $190.7 \pm 19.4$ & 0.56 \\
pH 4.00 & $127.5 \pm 12.1$ & 0.40 & $163.3 \pm 13.6$ & 0.52 \\
\hline
\end{tabular}

NSs = nanospheres; NCs = nanocapsules; PDI = polydispersity index.

\section{Morphological and dimensional analysis}

Morphological analysis revealed a spherical shape and a rather homogeneous distribution for both the NSs and NCs, with a modest state of aggregation and a high yield (Fig. 1). The core extraction process did not alter the morphology of the NCs as shown in Figures $1 \mathrm{~B}$ and $1 \mathrm{C}$.

Quantitative information regarding the dimensions of the NPs were supplied by DLS measurements on NS and NC samples. The analysis showed an average radius between $127.5 \pm$ 12.1 and $218.9 \pm 21.2 \mathrm{~nm}$ for NSs and a slight increase in NC dimensions with an average radius between $163.3 \pm 13.6$ and $214.7 \pm 19.7 \mathrm{~nm}$. Table I summarizes the average radius and PDI of the NSs and NCs dispersed in different solvents, highlighting the influence of the dispersant medium on the state of aggregation. In particular, a lower average radius was observed for the NP aggregates dispersed in buffered solution at $\mathrm{pH} 4.00$, in accordance with the molecular composition of the samples that caused an electrostatic repulsion. The PDI was 0.4 for NSs and 0.52 NCs suggesting that although the dispersant had a positive effect on the state of aggregation of the samples, some NPs aggregates were still present.

\section{FTIR chemical imaging analysis}

FTIR chemical imaging analysis highlighted the presence of the 3 components in the NPs. Figure 2 shows the chemical 
A

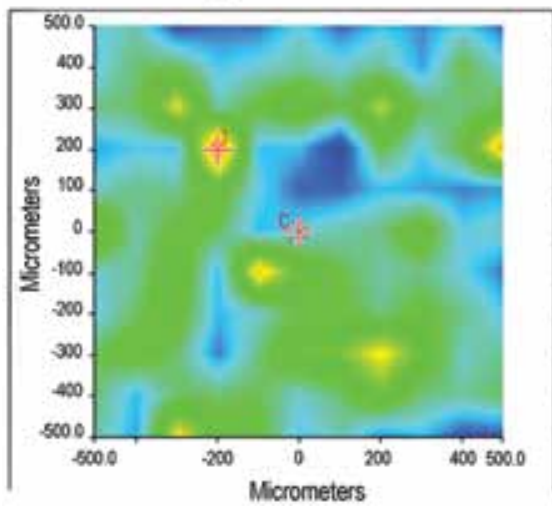

A

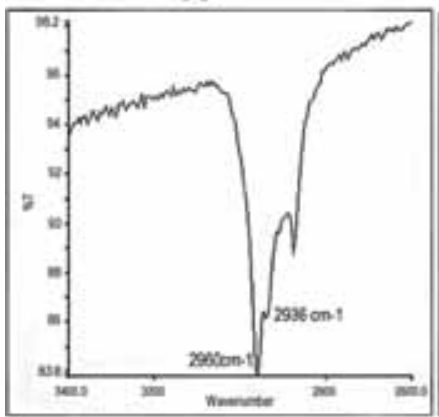

B

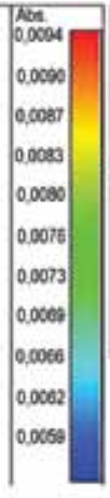

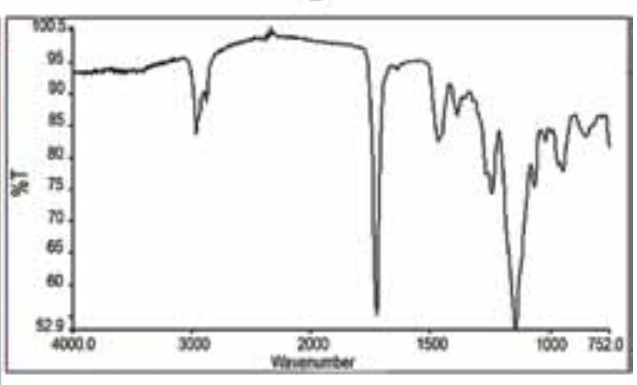

Fig. 2 - FT-IR Chemical Imaging analysis. (A) Chemical map and spectrum (B) of the terpolymer based NPs.

D

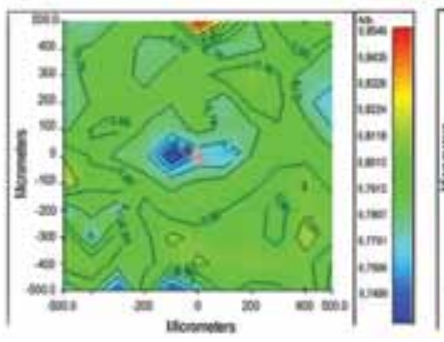

E

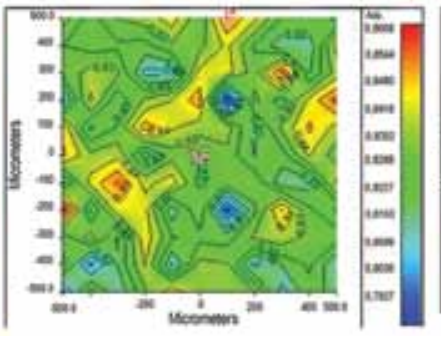

B

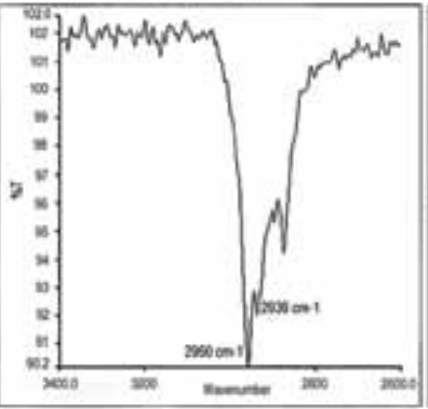

C

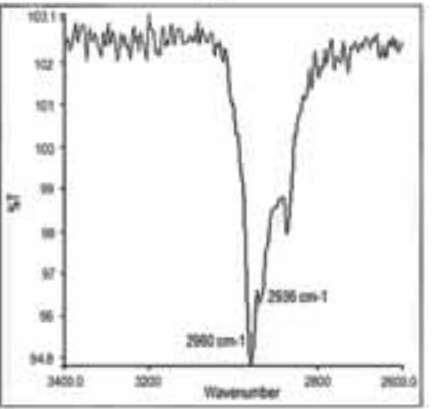

Fig. 3 - FT-IR Chemical Imaging analysis on the NPs. (A) NSs spectrum; (B) NCs spectrum before extraction and (C) after extraction; (D) Chemical map as a function of PMMA/PBMA for NSs, (E) NCs before and (F) after extraction.

map (Fig. 2A) and spectrum (Fig. 2B) acquired on terpolymerbased samples. Diagnostic bands associated with the $3 \mathrm{com}$ ponents are visible: the absorption related to $\mathrm{CxHy}$ groups between $3000-2700 \mathrm{~cm}^{-1}$, the high adsorption around 1700 $\mathrm{cm}^{-1}$ associated with the esters of the polymer matrix, a small band around $1680 \mathrm{~cm}^{-1}$ attributable to the $\mathrm{C}=\mathrm{C}$ bond of the PDMAEMA and (PEG)MEMA, and the adsorption related to the C-O-C group between $1200 \mathrm{~cm}^{-1}$ and $1000 \mathrm{~cm}^{-1}$. FTIR chemical imaging analysis was also carried out to assess the successful extraction of the core from the NCs. The possibility of evaluating the presence of PMMA is related to the adsorption of $\mathrm{CH}$ groups. Spectra related to the NSs and NCs before and after extraction were acquired (Figs. 3A, 3B, 3C). The NC spectrum before extraction showed the absorption around $2936 \mathrm{~cm}^{-1}$ related to the $\mathrm{CH}$ contribution of PMMA, and the adsorption around $2960 \mathrm{~cm}^{-1}$ related to the $\mathrm{CH}$ contribution of the PBMA (Fig. 3B). The maps as a function of the ratio PMMA/PBMA between 2930 to $2940 \mathrm{~cm}^{-1}$ and 2936 to $2953 \mathrm{~cm}^{-1}$ were acquired for NSs and NCs before and after extraction in order to investigate PMMA core removal (Figs. 3D, 3E, 3F). The band ratio of the NCs before extraction $(R=0.8-0.85)$ was higher than that of the NSs (0.75-0.80), confirming the presence of the PMMA core. After the extraction process, a decrement of the PMMA/PBMA ratio $(R=0.75-0.80)$ due to the reduction of the PMMA contribution was observed.

\section{Absorption test}

The encapsulation efficiency was evaluated by HPLC analysis of the absorption medium at the end of the test. Both the systems showed good encapsulation efficiency, reaching a value of $67.95 \%$ for the NSs and $87.1 \%$ for the NCs. The presence of the DNA was also investigated by FTIR chemical imaging analysis. Spectra acquired from the chemical map of the samples after adsorption showed the presence of a small band that fits on the adsorption of the ester group (data not shown). The second derivative spectra of the DNA showed a diagnostic 

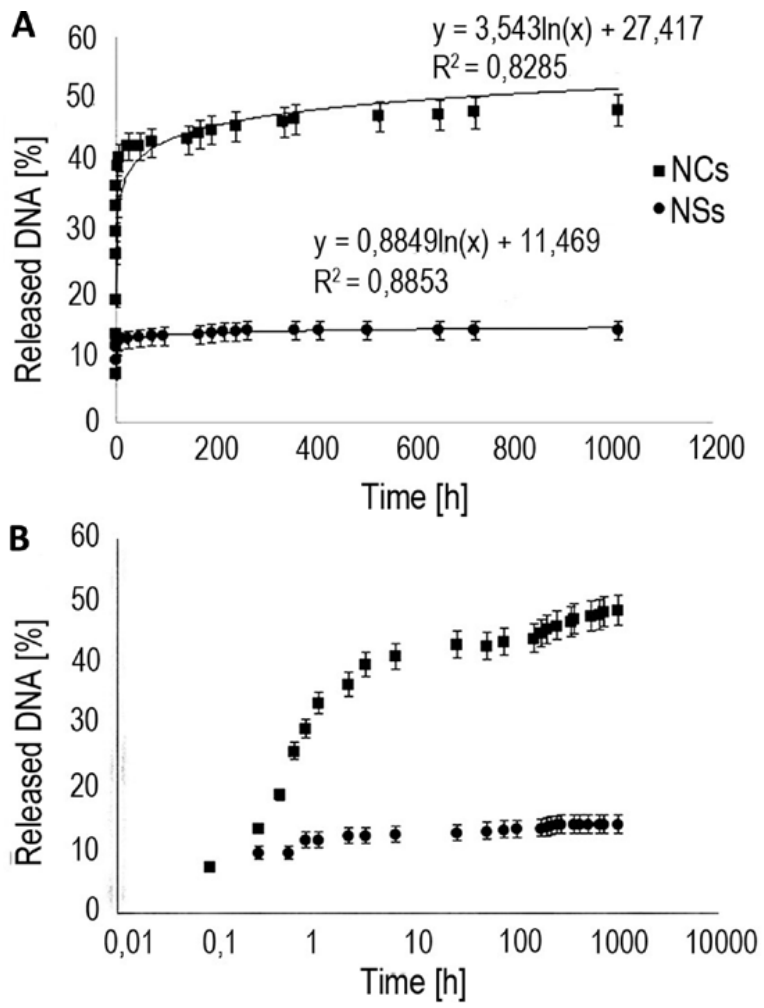

Fig. 4 - Drug delivery test. (A) Trend of DNA release from NPs, linear scale and (B) Log scale.

band around $1685 \mathrm{~cm}^{-1}$, related to the vibration of the carbonyl group of the DNA bases. The second derivative spectra of NSs and NCs, respectively, before absorption did not reveal the presence of the diagnostic band of the DNA. The band around $1685 \mathrm{~cm}^{-1}$ was evident once again in the second derivative spectra of the NPs after absorption, confirming the presence of the nucleic acid on the NPs.

\section{DNA release test}

The amount of released DNA from NPs was evaluated by HPLC analysis on the samples collected at predetermined intervals. The release kinetics are shown in Figures $4 \mathrm{~A}$ and $4 \mathrm{~B}$. In both cases, a rapid release in the first hours (burst effect) was observed, which tended to decrease over the following days. Moreover, HPLC analysis carried out on a solution of DNA in PBS (initial concentration of $1 \mathrm{mg} / \mathrm{ml}$ ) after ultracentrifugation procedure highlighted a concentration value similar to the theoretical one, with an error of \pm 0.005 that can be neglected, suggesting that the initial burst effect is due to the release of DNA absorbed onto NPs. DNA release from NCs was higher than the one from NSs and prolonged in time. The models shown in Figure 4 demonstrate a good correlation degree for both the NSs and the NCs with a coefficient $\mathrm{n}<0.50$.

\section{Cytotoxicity test}

The cytotoxicity of the produced NPs was preliminarily tested using the MTT cytotoxicity assay. NS and NC extracts were

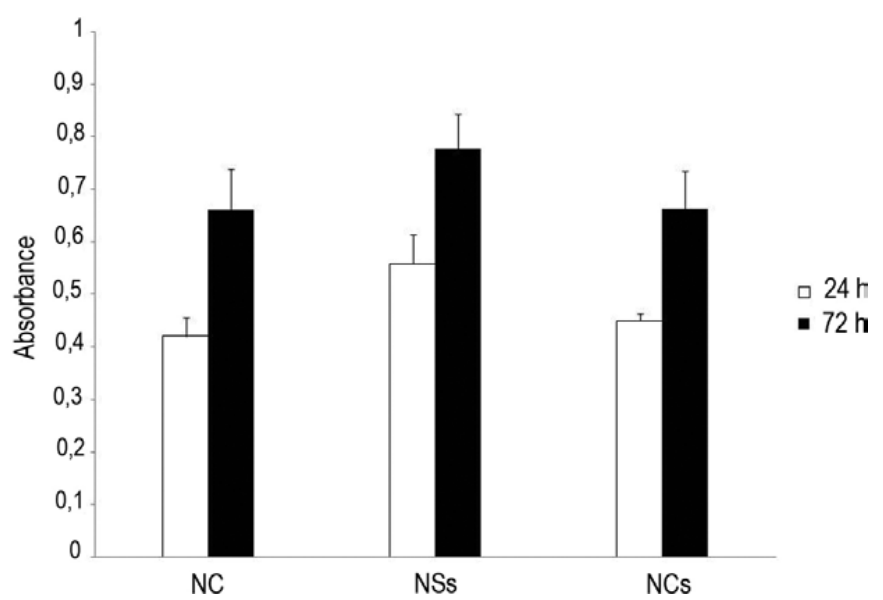

Fig. 5 - Cytotoxicity MTT test of NCs and NSs extracts after $24 \mathrm{~h}$ $(\square)$ and $72 \mathrm{~h}(\boldsymbol{\square})$ of incubation with $3 \mathrm{~T} 3$ cells. Comparison with the negative control is shown. The results are the mean $( \pm S D)$ of 10 measurements.

added to 3T3 cells and the viability of the cells was evaluated after exposure periods of $24 \mathrm{~h}$ and $72 \mathrm{~h}$, respectively. The obtained results are shown in Figure 5 . It can be observed that at both $24 \mathrm{~h}$ and $72 \mathrm{~h}$ the mitochondrial activity of the cells was not affected by the extracts. No significant differences were found between treated and control wells ( $F$-test, $P>.05)$ with the exception of NCs extracts after $24 \mathrm{~h}$ of incubation $(P<.05)$.

\section{Discussion and conclusions}

The combination of radical polymerization of 3 distinct monomers by the precipitation method and core-shell procedure via template method allowed the production of new hollow particles with a spherical shape and nanometric dimensions.

FTIR chemical imaging analysis confirmed the successful copolymerization both for NSs and NCs. In view of a possible use of these nano-systems for gene therapy, DNA was selected as the model molecule to be loaded into NPs. Both systems showed good absorption efficiency, probably due to the presence of PDMAEMA, which provides amino groups to the terpolymer, thus improving the molecular interaction between NPs and DNA functional groups.

Moreover, the results of the absorption test demonstrated the effect of the hollow structure in maximizing the loading of nucleic acid, as shown by an evident increase in the amount of DNA encapsulated into the NCs with respect to the NSs. This behavior was also reflected on the release kinetics, which showed an amount of delivered DNA from the NCs greater than that from NSs. Both systems highlighted the typical kinetics of release from NPs (21-23). Analyzing the delivery profiles more in depth (Fig. 4B), especially in the case of NCs, the release kinetics can be divided into 3 steps. In a very early stage a slower release occurred, due to the fact that the system tends to achieve equilibrium with the surrounding environment. In a second phase a burst effect was evident, probably due to the DNA weakly bound to the surface of the NCs and/or to the matrix immediately below 
the NC surface. The third phase showed a sustained and prolonged release, due to the DNA located into the NCs cavity and its diffusion through the polymeric matrix that allows a slower and more prolonged release than the one from NSs. After 20 days the absorbed DNA was not completely released and the steady state was not reached for the NCs. This could be due to the fact that there was DNA remaining inside the cavity that probably need much more time to diffuse and be delivered. Regarding NSs, the steady state was reached after 9 days, but in this case as well not all the absorbed DNA was released, probably because of the electrostatic interactions occurring between the terpolymer and the functional groups of the nucleic acid. The release order value $(n<0.50)$ obtained for both the samples using the semi empirical model of Peppas (20) is typical of a release dominated by a purely diffusive mechanism, in which there is no influence due to macromolecular relaxation phenomena, as expected in the case of a cross-linked structure. Finally, a preliminary cytotoxicity investigation showed no toxic effects of the NPs on the cells, thus supporting the potential use of these particles as carriers for drug delivery applications.

In conclusion, the results obtained in this study showed that thanks to their chemical composition, terpolymer-based NPs may be suitable systems to promote interaction with oligonucleotidic drugs, thus obtaining a carrier for the delivery of nucleic acid. The possibility of obtaining a hollow structure allows the amount of the absorbed DNA to be maximized and a more prolonged release kinetics than those from NSs. The hollow structure allows offer the advantage of allowing a higher amount of drug to be administrated using a lower amount of polymer.

The preliminary results obtained in this work encourage us to continue in the evaluation of these new polymeric nanocarriers as DNA delivery systems in view, of a possible application in gene therapy for cancer treatment once suitable modifications have been made.

\section{Acknowledgement}

Andrea Baldassare is kindly acknowledged for cytotoxicity test support in this work.

\section{Disclosures}

Financial support: This work was supported by Institutional funds of CNR.

Conflict of interest: The authors declare no conflict of interest.

Meeting presentation: The data were presented at the National Congress of the Italian Biomaterial Society (SIB), July 2-4, 2014, Palermo, Italy.

\section{References}

1. Jia F, Liu X, Li L, Mallapragada S, Narasimhan B, Wang Q. Multifunctional nanoparticles for targeted delivery of immune activating and cancer therapeutic agents. J Control Release. 2013; 172(3):1020-1034.

2. Eidi $H$, Joubert $O$, Némos $C$, et al. Drug delivery by polymeric nanoparticles induces autophagy in macrophages. Int J Pharm. 2012;422(1-2):495-503.
3. Danhier F, Ansorena E, Silva JM, Coco R, Le Breton A, Préat V. PLGA-based nanoparticles: an overview of biomedical applications. J Control Release. 2012;161(2):505-522.

4. Torchilin VP. Drug targeting. Eur J Pharm Sci. 2000;11(Suppl 2):S81-S91.

5. Stylios GK, Giannoudis PV, Wan T. Applications of nanotechnologies in medical practice. Injury. 2005;36(Suppl 4):S6-S13.

6. Cheng R, Meng F, Deng C, Klok HA, Zhong Z. Dual and multistimuli responsive polymeric nanoparticles for programmed sitespecific drug delivery. Biomaterials. 2013;34(14):3647-3657.

7. Fu GD, Li GL, Neoh KG, Kang ET. Hollow polymeric nanostructures - Synthesis, morphology and function. Prog Polym Sci. 2011;36(1):127-167.

8. Wang W, Luo C, Shao S, Zhou S. Chitosan hollow nanospheres fabricated from biodegradable poly-D,L-lactide-poly(ethylene glycol) nanoparticle templates. Eur J Pharm Biopharm. 2010; 76(3):376-383.

9. Han J, Song G, Guo R. Fabrication of polymer hollow nanospheres by a swelling evaporation strategy. J Polym Sci A. 2007; 45(13):2638-2645.

10. Han J, Song G, Guo R. A facile solution route for polymeric hollow spheres with controllable size. Adv Mater. 2006;18(23): 3140-3144.

11. Chu H, Liu N, Wang X, Jiao Z, Chen Z. Morphology and in vitro release kinetics of drug-loaded micelles based on well-defined PMPC-b-PBMA copolymer. Int J Pharm. 2009;371(1-2):190-196.

12. Luo YL, Zhang LL, Xu F. Synthesis, micellization and caffeine drug release kinetics of novel PBMA-b-PNIPAAm block polymer brushes. Chem Eng J. 2012;189-190:431-442.

13. Xu FJ, Yang WT. Polymer vectors via controlled/living radical polymerization for gene delivery. Prog Polym Sci. 2011;36(9): 1099-1131.

14. Mathew A, Cao H, Collin E, Wang W, Pandit A. Hyperbranched PEGmethacrylate linear pDMAEMA block copolymer as an efficient non-viral gene delivery vector. Int J Pharm. 2012;434 (1-2):99-105.

15. Kundu PP, Sharma V. Synthetic polymeric vectors in gene therapy. Curr Opin Solid State Mater Sci. 2008;12(5-6):89-102.

16. Agarwal S, Zhang Y, Maji S, Greiner A. PDMAEMA based gene delivery materials. Mater Today. 2012;15(9):388-393.

17. Mathaes R, Winter G, Besheer A, Engert J. Influence of particle geometry and PEGylation on phagocytosis of particulate carriers. Int J Pharm. 2014;465(1-2):159-164.

18. Tao Y, Han J, Dou H. Surface modification of paclitaxel-loaded polymeric nanoparticles: Evaluation of in vitro cellular behavior and in vivo pharmacokinetic. Polymer (Guildf). 2012; 53(22):5078-5086.

19. Vroman B, Ferreira I, Jérôme $C$, Jérôme R, Préat V. PEGylated quaternized copolymer/DNA complexes for gene delivery. Int J Pharm. 2007;344(1-2):88-95.

20. Siepmann J, Peppas NA. Modeling of drug release from delivery systems based on hydroxypropyl methylcellulose (HPMC). Adv Drug Deliv Rev. 2012;64:163-174.

21. Soppimath KS, Aminabhavi TM, Kulkarni AR, Rudzinski WE. Biodegradable polymeric nanoparticles as drug delivery devices. J Control Release. 2001;70(1-2):1-20.

22. Bazylinska U, Lewinska A, Lamch L, Wilk KA. Polymeric nanocapsules and nanospheres for encapsulation and longsustained release of hydrophobic cyanine-type photosensitizer. Colloid Surf., A. 2014;442:42-49.

23. Vafayi L, Gharibe S. Investigation of In Vitro Drug Release from Porous Hollow Silica Nanospheres Prepared of ZnS@SiO 2 CoreShell. Bioinorg Chem Appl. 2013;2013:541030. 